\title{
SPECIAL THEORY OF RELATIVITY: A RETELL
}

\author{
by MANGHAT SRIKUMAR
}

\begin{abstract}
In this paper Einstein's special theory of relativity is revisited and a new facet implied by the theory is discovered. It is shown that this newly discovered facet has far reaching consequences by using it to find a solution/explanation to some of the most outstanding problems in contemporary physics. As he goes through the theory presented here it will become apparent to the reader that the theory sheds a new light on the most established concepts in physics while paving the way towards newer inquiries into the unknown.
\end{abstract}

Keywords: Relativity, dark matter, dark energy, black holes, grand unified theory, electrodynamics.

\section{I.INTRODUCTION}

Consider the following outstanding problems in contemporary physics:

a) It has been observed by astrophysicists that celestial bodies in certain galaxies were under the influence of gravitational forces that could not be produced by conventional masses alone in the galaxies. It has been conjectured that dark matter [1][2] were responsible for the extra forces but physicists have not been able to prove the existence of these masses till date.

b) It has been observed that the rate of expansion of the universe differs slightly from the rate predicted by Einstein's general theory of relativity. It has been conjectured that dark energy [3] was responsible for the difference in rates but physicists have not been able to prove the existence of this energy till date.

c) Some stars are known to die as black holes [4] where gravitation force is the overpowering force in action. No one knows till date the fate of black holes.

d) Grand unified theories [5] have been proposed in the literature that unifies three of the four basic forces in nature viz.; strong nuclear force, weak nuclear force and electromagnetic force. But physicists have hitherto failed to conjecture any relation between the fourth basic force, gravitation, to any of the other forces.

e) From the theory of electrodynamics [6][7] we know that accelerating electric charges emit electromagnetic radiation. But it is a well-known and unsolved problem that the formula for the loss of kinetic energy of the body due to radiation does not equal the formula for the energy of the radiation emitted.

Can a single theory address these problems and provide an explanation/solution? The proposed paper attempts to provide such a theory. The paper starts from Einstein's special theory of relativity and 
proceeds to discover an undisclosed facet that the theory implies. In the light of this new discovery, the problems stated above are then addressed one by one.

\section{II.EINSTEIN'S SPECIAL THEORY OF RELATIVITY IN BRIEF}

Let us consider Einstein's special theory of relativity [8][9][10] in brief. The explanation of the theory begins from the theoretical finding by $\mathrm{H}$. A. Lorentz in a seminal paper in 1825 that the speed of light in vacuum is the same irrespective of the speed of the body in which it is measured. In order to explain the theory, let us consider two bodies - an embankment at rest and a train which is moving with a speed $\mathbf{v}$ with respect to the embankment. Let the speed of light be $\mathrm{c}$ with respect to the fixed embankment. Then, common sense would dictate that the speed of light measured in the frame of reference fixed with respect to the train would be c-v. But as proved by Lorentz, the speed of light with respect to the moving train is also $\mathbf{c}$. Einstein explained this contradiction by stating that the measure of time and the measure of distance in the direction of movement is different for a fixed body and for a body moving with a speed with respect to the fixed body.

Let $\mathbf{x}, \mathbf{y}$ and $\mathbf{z}$ be the distance Cartesian coordinates and $\mathbf{t}$ be the time coordinate of any event with respect to the fixed body and $\mathbf{x}^{\prime}, \mathbf{y}^{\prime}$ and $\mathbf{z}^{\prime}$ and $\mathbf{t}^{\prime}$ be the respective coordinates of the event with respect to a body moving with a speed $\mathbf{v}$ with respect to the fixed body along $\mathbf{x}\left(\mathbf{x}^{\prime}\right)$ axis. See figure 1 below:

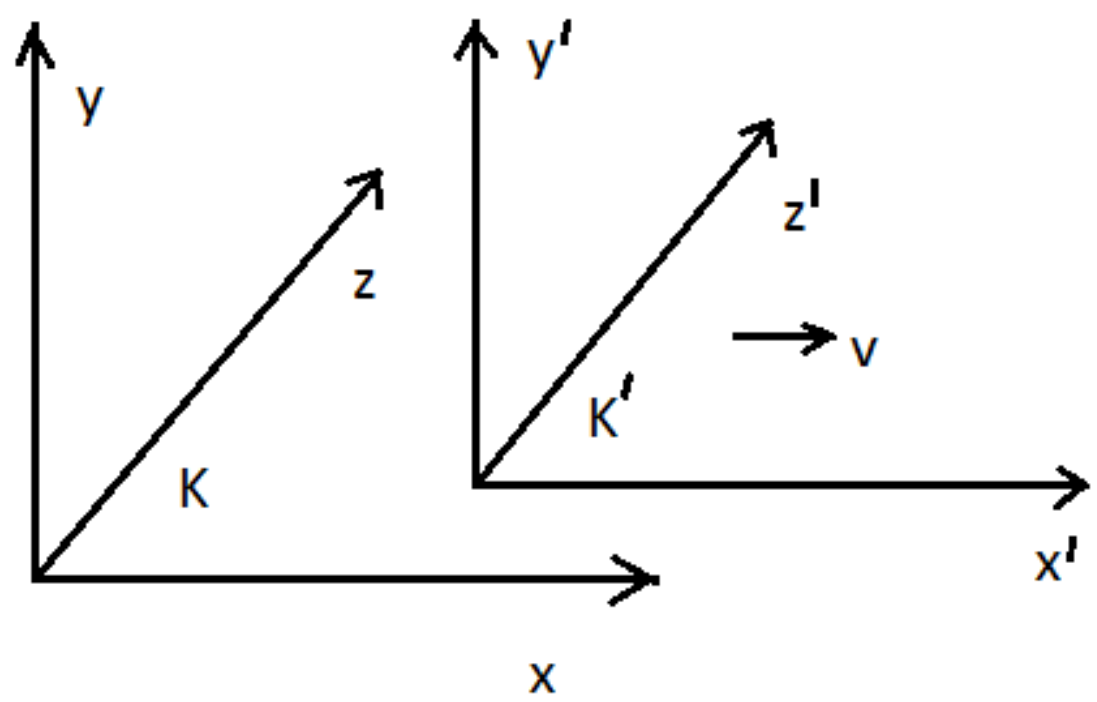

Figure 1: The coordinate systems

So, in order that the speed of light be the same in the frames of references with respect to the each of the two bodies, Einstein showed that, the time and distance coordinates of the bodies need to be related to each other by the so-called Lorentz transformation as follows: 


$$
\begin{gathered}
x^{\prime}=\frac{x-v t}{\sqrt{1-\frac{v^{2}}{c^{2}}}} \\
y^{\prime}=y \\
z^{\prime}=z \\
t^{\prime}=\frac{t-\frac{v}{c^{2}} x}{\sqrt{1-\frac{v^{2}}{c^{2}}}}
\end{gathered}
$$

\section{III.LENGTH OF A METER ROD IN DIFFERENT REFERENCE FRAMES}

Let us call the fixed $\mathbf{x}, \mathbf{y}, \mathbf{z}, \mathbf{t}$ coordinate system $\mathbf{K}$ and the moving $\mathbf{x}^{\prime}, \mathbf{y}^{\prime}, \mathbf{z}^{\prime}, \mathbf{t}^{\prime}$ coordinate system $\mathbf{K}^{\prime}$. If we place a meter-rod in the $\mathbf{x}^{\prime}$-axis of $\mathbf{K}^{\prime}$ in such a manner that one end coincides with the point $\mathbf{x}^{\prime}=0$, whilst the other end coincides with the point $\mathbf{x}^{\prime}=1$, what is the length of the meter-rod relatively to the system $\mathbf{K}$ ? In order to learn this, we need only ask where the beginning of the rod and the end of the rod lie with respect to $\mathbf{K}$ at a particular time $\mathbf{t}$ of the system $\mathbf{K}$. By means of the first equation of the Lorentz transformation the values of these two points at the time $t=0$ can be shown to be

$$
\begin{gathered}
x_{(\text {begining of rod })}=0 . \sqrt{1-\frac{v^{2}}{c^{2}}} \\
x_{(\text {end of rod })}=1 . \sqrt{1-\frac{v^{2}}{c^{2}}}
\end{gathered}
$$

the distance between the points being $\sqrt{1-\frac{v^{2}}{c^{2}}}$. But the meter-rod is moving with the speed $\mathbf{v}$ relative to K. It therefore follows that the length of a rigid meter-rod moving in the direction of its length with a speed $\mathbf{v}$ is $\sqrt{1-\frac{v^{2}}{c^{2}}}$ of a meter. Thus, the measure of length in the direction of movement decreases by factor of $\sqrt{1-\frac{v^{2}}{c^{2}}}$ as speed of the body increases by $\mathbf{v}$.

\section{IV.A NEW FACET EMERGING FROM THE SPECIAL THEORY}

In order to get a new insight into the special theory of relativity we make the following hypotheses: 
1. The length of a body is not a constant but varies with its speed.

2. The length of the body decreases by a factor of $\sqrt{1-v^{2} / c^{2}}$ in all directions as the speed increases to $\mathbf{v}$ from rest.

Going by these hypotheses, 1 meter of the stationary body is equivalent to $\sqrt{1-v^{2} / c^{2}}$ meters of the body moving with speed $\mathbf{v}$ (measured with respect to a stationary frame of reference). So, what is the length of the body as measured in the frame of reference fixed with respect to the body in motion? Considering the hypotheses stated above alone, one possible formulation is that 1 meter of the body measured in a frame of reference of the body (whether at rest or in motion) is equal to $\sqrt{1-v^{2} / c^{2}}$ meters of the body with respect to a stationary frame of reference so that when the speed of the body is zero the length measured in the frame of reference of the body is equal to the length as measured from the stationary frame. From the special theory of relativity as explained in previous sections we know that this is indeed true in the direction of movement as the measure of length reduces by a factor of $\sqrt{1-v^{2} / c^{2}}$ in the direction of movement as the speed of the body increases to $\mathbf{v}$ from rest. The above mentioned hypotheses now make sense as there is now a physical basis to the proposition that the measure of length reduces with speed. Also, a simple solution/explanation to many unsolved problems in contemporary physics is afforded by assuming that the hypotheses are true as we shall soon see.

We shall assume that the hypotheses are true for the treatment in the rest of the paper. (Note: There appears to be a violation of the law of conservation of energy as the dimension of the body seems to be reducing of its own without any agency acting on the body for that purpose: this problem is addressed in section VIII of the paper).

So, we have that as a body gains speed its physical dimensions shrink. If $\mathbf{L}$ is initial length after gaining a speed of $\mathbf{v}$ its new length $L^{\prime}$ is

$$
L^{\prime}=L \sqrt{1-v^{2} / c^{2}}
$$

In common observation these changes in dimension of a body are not immediately apparent as either the changes in speed encountered in nature are small compared to the speed of light or the dimensions of the body that have considerable speeds are so small that changes in dimension are not measurable in practice.

Since there is no direct involvement of the accelerating agency in reducing the dimensions of the accelerated body, intuitively, the following statement can be made regarding the relation between the dimension of a body and its kinetic energy: 


\section{The dimension of a body and its kinetic energy are intimately related: Increase (decrease) of one causes a decrease (increase) in the other.}

\section{V.ACCELERATION OF MASSES TOWARDS EACH OTHER}

The following facts can be deduced from equation (1):

a) A body at rest or moving with uniform speed does not experience any length shortening effect. It is only when the speed of the body increases or, in other words, when the body accelerates, that the points on the body tend to move towards or are attracted towards each other.

b) When a body accelerates from rest to a speed of c, it loses all its dimensions (dimension becomes 0 ) irrespective of the initial dimension of the body. Suppose two bodies of unequal length are accelerated together from rest to speed $\mathbf{c}$ within time $\mathbf{t}$. Equation (1) says that both the bodies lose their dimensions within the same time $t$. Thus, the time taken for bodies to lose their dimensions is dependent only on the acceleration of the bodies.

Also, the following points are obvious and need to be noted:

A. As per equation (1), the acceleration that the point on the body experiences as it shrinks towards the center of convergence is dependent on the acceleration of the body; greater the acceleration of the body, greater the acceleration that the point experiences towards the center of convergence and conversely.

B. The deceleration that the point experiences as it shrinks towards the center of convergence is solely due to the density of mass within the body: Greater the density, greater the hindrances that point faces in shrinking towards the center of convergence, greater the deceleration of the point.

Consider a spherical body of mass $\mathbf{M}$ and radius $\mathbf{R}$ with a point $\mathbf{A}$ on the surface. For simplicity of understanding we shall assume that the body has uniform density everywhere so that whatever be the rate at which the dimension of the body is shrinking, it is equal in all directions and is thus directed towards the center of the body. For such a body the radius at rest $\mathbf{R}$ and the radius $\mathbf{R}^{\prime}$ after gaining a speed $\mathbf{v}$ is related as:

$$
R^{\prime}=R \sqrt{1-v^{2} / c^{2}}
$$

Let us find the time taken for the body to lose dimensions as the body accelerates from rest to speed $\mathbf{c}$ with a constant acceleration a. In other words, let us find the time taken for point $A$ to shrink to the centre of the body as the body accelerates to speed $\mathbf{c}$ from rest. 
Since only the mass $\mathbf{M}$, radius $\mathbf{R}$ and acceleration a terms are involved, let us formulate the following equation for the acceleration (see remark $A$ above) $\boldsymbol{\alpha}$ of point $A$ towards the center:

$$
\alpha=k(a) \frac{M}{R^{2}}
$$

, where $\mathbf{k}(\mathbf{a})$ is a function of only the acceleration $\mathbf{a}$ of the body and increasing with it.

If the time taken for the dimension to shrink to 0 deduced using this equation is found to be dependent only on the acceleration a of the body, then we can say that the formulation of this equation for the acceleration of point $A$ is correct.

Let us proceed to find out the time taken for shrinking.

Let us assume that the sphere is uniformly accelerating from 0 to $\mathbf{c}$ with an acceleration $\mathbf{a}$. Let us choose a coordinate system such the center of the sphere is at the origin of the system and point $A$ lies along any of the coordinate axes (refer to figure 2). From equation (3), representing by $\mathbf{R}(\mathbf{t}$ ) the distance of point $A$ from the origin at time $t$, the acceleration $\alpha$ of point $A$ is

$$
\alpha=\frac{d^{2} R(t)}{d t^{2}}=(-) k(a) \frac{M}{R(t)^{2}}
$$

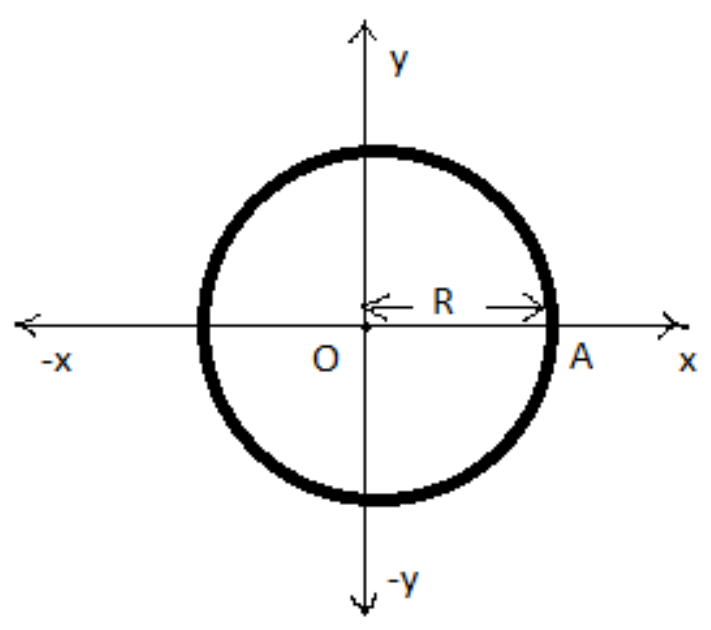

Figure 2: A sphere of radius $\mathbf{R}$

The equation above gives the acceleration of a point on the surface of the sphere with radius $\mathbf{R}(\mathbf{t})$ when it is freely moving towards the center as the body accelerates. But actually, its motion is opposed by the mass particles in the space between the sphere of radius $\mathbf{R}(\mathbf{t})$ and the center (see remark $B$ in the beginning of this section) - greater the density of the mass particles lesser the acceleration of the point. To account for the opposition mass particles provide to the motion of the point on the surface, we multiply the right hand side of the equation with a factor $\lambda$ that is inversely proportional to the average density of the matter in the region between radius $\mathbf{R}(\mathbf{t})$ and the center. Thus, $\boldsymbol{\lambda}$ is 


$$
\lambda=\frac{B \cdot \frac{4}{3} \pi R(t)^{3}}{M}
$$

where $\mathbf{B}$ is a proportionality constant.

Thus we have

$$
\alpha=\frac{d^{2} R(t)}{d t^{2}}=(-) k(a) \frac{M}{R(t)^{2}} \cdot \lambda
$$

Or putting the value of $\lambda$, we have

$$
\frac{d^{2} R(t)}{d t^{2}}=(-) k(a) \frac{M}{R(t)^{2}} \cdot \frac{B \cdot \frac{4}{3} \pi R(t)^{3}}{M}
$$

Or,

$$
\frac{d^{2} R(t)}{d t^{2}}=(-) \frac{4 \pi B k(a) R(t)}{3}
$$

Now, when a body accelerates, it does not attain the speed of light and does not lose its entire dimension, because then mass would disappear (more on this in section VII). Therefore, the dimension of the sphere within the radius, say $\mathbf{r}_{\mathbf{0}}$, is never lost (see Figure 3 ) due to acceleration.

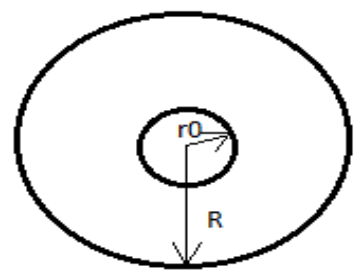

Figure 3: Dimension $r_{0}$ not lost due to acceleration.

But we need to find the time it takes for the hypothetical case in which the dimension reduces to 0 as the body accelerates from rest to the speed of light in order to prove that the time taken is independent of the initial dimension of the body.

This time taken for the point to accelerate from point $A$ at a radius of $\mathbf{R}(\mathbf{t})$ to the center $\mathbf{O}$ of the sphere, is obtained by finding a solution to the equation above which can be found by many methods, out of which one method involves taking the Laplace transform of each term in the above equation (Note: Laplace transform of $\mathbf{R}(\mathbf{t})$ exists since $\mathbf{R}(\mathbf{t})$ is obviously integrable over a finite interval $\mathbf{t}_{1}<\mathbf{t}<\mathbf{t}_{2}$, where 0 
$\leq \mathbf{t}_{1}<\mathbf{t}_{2}<\infty$ and $\lim _{t \rightarrow \infty} e^{-\sigma t}|\boldsymbol{R}(\boldsymbol{t})|$ exists for some value of $\sigma---$ the conditions required by the definition of Laplace transforms). Taking the Laplace transform for each term in the above equation we have:

$$
\boldsymbol{s}^{2} \boldsymbol{R}(\boldsymbol{s})-\boldsymbol{s} \boldsymbol{R}\left(0^{-}\right)-\boldsymbol{R}^{\prime}\left(0^{-}\right)=(-) \frac{4 \pi \boldsymbol{B} \boldsymbol{k}(\boldsymbol{a}) \boldsymbol{R}(\boldsymbol{s})}{3}
$$

where $\mathbf{s}$ is the complex frequency, $\boldsymbol{R}\left(0^{-}\right)$is the value of $\mathbf{R}(\mathbf{t})$ at $\mathbf{t}=0^{-}$(i.e; just before $\mathbf{t}=0$ ) and $\boldsymbol{R}^{\prime}\left(0^{-}\right)$is the value of the first derivative of $\mathbf{R}(\mathbf{t})$ at $\mathbf{t}=0^{-}$. Since, we know that $\boldsymbol{R}\left(0^{-}\right)=R$ and $\boldsymbol{R}^{\prime}\left(0^{-}\right)=0$ (since speed of point $A$ is zero just before acceleration of the body starts), we have

$$
\boldsymbol{s}^{2} \boldsymbol{R}(\boldsymbol{s})-\boldsymbol{s} R=(-) \frac{4 \pi \boldsymbol{B} \boldsymbol{k}(\boldsymbol{a}) \boldsymbol{R}(\boldsymbol{s})}{3}
$$

Or,

$$
\boldsymbol{R}(\boldsymbol{s})=\frac{R \boldsymbol{s}}{\boldsymbol{s}^{2}+\frac{4 \pi \boldsymbol{B} \boldsymbol{k}(\boldsymbol{a})}{3}}
$$

Taking inverse Laplace transform of the terms we have

$$
R(t)=R \cos \left(\sqrt{\frac{4 \pi B k(a)}{3}} \cdot t\right) u(t)
$$

where $\mathbf{u}(\mathbf{t})$ is a unit step function which has a value equal to unity for $\mathbf{t}>0$ and is equal to 0 for $\mathbf{t}<0$.

The time period $T_{0}$ of oscillation of point $A$ about the center is thus

$$
\boldsymbol{T}_{\boldsymbol{o}}=\frac{2 \pi}{\sqrt{\frac{4 \pi \boldsymbol{B} \boldsymbol{k}(\boldsymbol{a})}{3}}}
$$

Since mass would disappear completely as the point A reaches the center, the actual time taken for the point $A$ to reach the center is $1 / 4^{\text {th }}$ of this period. The time taken is thus

$$
T=\frac{T_{o}}{4}=\sqrt{\frac{3 \pi}{16 B k(a)}}
$$

Thus, the time taken is dependent only on the acceleration a of the body and is independent of the initial dimension of the body in line with what was found using Einstein's equations. Hence, the formulation (3) for the acceleration of a point on the body is correct. 


\section{VI.OBSERVATIONS SO FAR}

The equation (3) has a resemblance to the gravitational equation for the acceleration of a body under the influence of a mass $\mathbf{M}$ at a distance of $\mathbf{R}$

$$
\alpha=G \frac{M}{R^{2}}
$$

where $\mathbf{G}$ is the gravitational constant.

We are led into the conclusion that Equation (3) is indeed the general form of the gravitational equation and that the gravitational effect is due to the acceleration of the system in which the bodies are situated. As the gravitational equation in the form stated above is known to be true throughout the realms of our galaxy, this system for us is presumably our galaxy itself. Thus, the gravitational effect that we experience is due to the acceleration of our galaxy and for our galaxy the value of $\mathbf{k}(\mathbf{a})$ is $\mathbf{G}$. In another galaxy, accelerating at a different rate, the value of $\mathbf{k}(\mathbf{a})$ and thus the gravitational attraction would be different.

This new insight provides the explanation for the concept of dark matter. Dark matters were conjectured as existing when the forces exerted in certain galaxies on various celestial bodies could not be explained by the observable masses of the galaxies. In the light of the above explanations it is clear that the forces itself were wrongly calculated since the gravitational constant used were itself incorrect.

Also, general theory of relativity [9] assumes a unique value $\mathbf{G}$ for the gravitational constant and predicts the rate of expansion of the universe on this basis. With multiple values of gravitational constant possible in the universe as explained in the proposed theory, there is little wonder that the prediction of the rate of expansion by the general theory is erroneous. The need for dark energy for explaining the observed rate is thus eliminated.

\section{VII.INSIGHTS FROM THE KINETIC ENERGY EQUATION}

The kinetic energy equation that Einstein derived in his theory is true even now and is as shown below:

$$
\boldsymbol{K} \cdot \boldsymbol{E}=\frac{\boldsymbol{m c ^ { 2 }}}{\sqrt{1-\frac{v^{2}}{c^{2}}}}
$$

Developing the expression in the form of a series:

$$
K . E=m c^{2}+\boldsymbol{m} \frac{v^{2}}{2}+\frac{3}{8} \boldsymbol{m} \frac{v^{4}}{c^{2}}+\cdots
$$


In the light of the above discussion, the kinetic energy equation can be interpreted as follows: the terms in the equation with the parameter $\mathbf{v}$ corresponds to the dimension of the body (let us call it the potent dimension) which were lost due to acceleration, whereas the term $\mathbf{m c}^{2}$ refers to that dimension of the body (let us call it the latent dimension) which is never lost due to acceleration. This means that after the body has reached a maximum speed, the dimension that the body is left with, contains the basic building blocks of mass that do not shrink further. These as yet undiscovered basic building blocks are such that they interact with each other creating larger dimensions and the various forms of matter that we see around us. The situation is illustrated in figure 4 as shown below assuming for simplicity of understanding that the basic building blocks of mass are all of the same type:

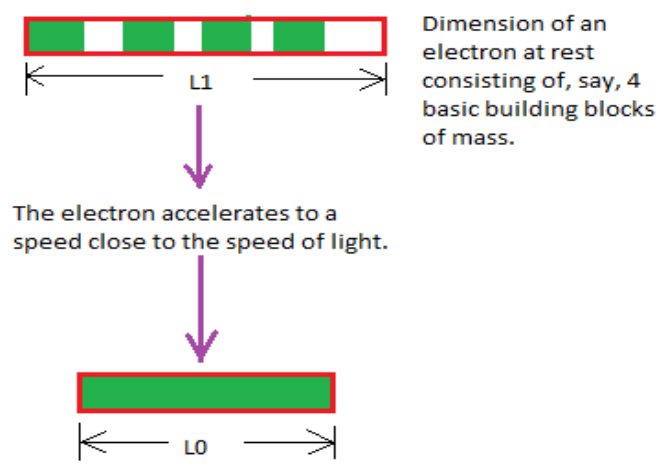

Dimension Lo corresponding to energy $\mathrm{E}=\mathrm{mc}$, is not lost. Dimension (L1-LO) is lost due to acceleration and the gaps between the basic mass building blocks disappear but the dimension of the blocks themselves does not shrink.

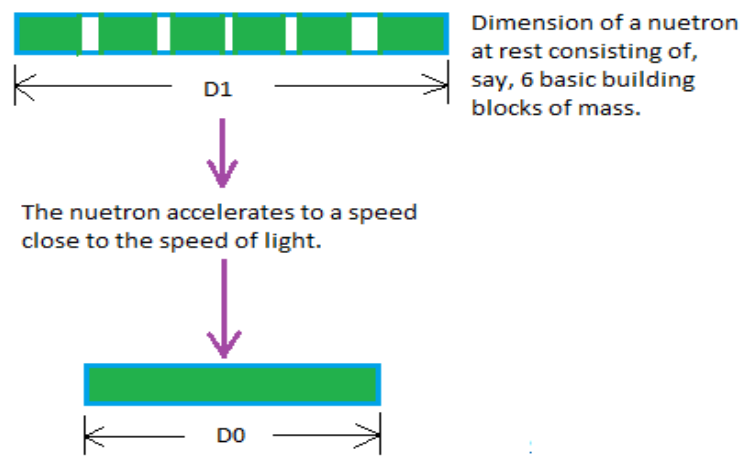

Dimension D0 corresponding to an energy $\mathrm{E}=\mathrm{mc}^{2}$, is not lost. Dimension (D1-D0) is lost due to acceleration and the gaps between the basic mass building blocks disappear but the dimension of the blocks themselves does not shrink.

Figure 4: Illustration of how the basic building blocks of mass form various entities and how the dimension is lost due to acceleration.

As shown above the latent dimension corresponding to energy $\mathbf{m c}^{2}$ is never lost by acceleration. The first example of a situation where this energy is lost is shown below:

Black Holes: The only force acting in a black hole is the unchecked inward gravitational pull (there is no opposition due to density of matter). In this case, the pressure developing at the center of the black hole acts directly on the matter there without the intervention of any opposing force. Therefore the dimension of the matter there (including the latent dimension) cannot be sustained in the present 3dimensional space system. So, as the matter is compressed and as energy associated with its dimensions cannot be lost, the matter is presumably released into another dimensional system or in other words into another universe. Thus, we have that black hole centers are passages to another dimensional system. Since it is like draining an ocean through a hole the size of a tip of a pin, the matter in the black holes disappear only very slowly into another dimensional space. 


\section{VIII.ROLE OF ELECTROMAGNETIC ENERGY}

I make the following proposition regarding the role of electromagnetic (EM) energy in the proposed theory with a promise of proving the statement shortly:

When a body moving with a constant speed absorbs electromagnetic energy its kinetic energy decreases and the decrease in kinetic energy is exactly equal to the electromagnetic energy absorbed. Similarly, when a body moving with constant speed releases electromagnetic energy its kinetic energy increases and the increase in kinetic energy is exactly equal to the electromagnetic energy released. (Note: These statements are reasonable and, as will be proved shortly, are right because the body, which is moving with constant speed, is not under the influence of any net force, and hence the total energy possessed by the body - sum of both kinetic and other energies - before the emission/absorption of electromagnetic energy is equal to the total energy of the body after the emission/absorption).

In order to prove the above statement, we would have preferred an experimental setup where a particle is subjected to electromagnetic waves and accelerating forces. But fortunately, we already have access to such situations as explained below:

a) It is found that in a hydrogen atom after absorption of EM wave, the kinetic energy difference of the electron in orbit in its neutral state and in its exited state is exactly equal to the energy of the EM wave absorbed. For example, kinetic energy of the electron in orbit around the nucleus of the hydrogen atom in its neutral state is $2.180 \times 10^{-18} \mathrm{~J}$. After absorption of an EM wave of $122 \mathrm{~nm}$, the electron moves into a higher orbit of the exited state where its kinetic energy is $5.449 \times 10^{-19} \mathrm{~J}$. It can be seen that the difference in kinetic energies of the two states is exactly equal to the energy of the EM waves absorbed, i.e; $1.630 \times 10^{-18} \mathrm{~J}$. This fact can also be observed for higher orbital jumps of the hydrogen electron.

The facts stated above cannot be explained in any other way but as follows: The electron absorbs EM wave and the mass carrying entity increases in dimension. The increase in dimension is followed by a reduction in speed and thus a decrease in the kinetic energy of the electron, the decrease being exactly equal to the energy of EM wave absorbed. The higher orbit is attained by the electron due to forces within the atom.

b) From the theory of electrodynamics we already know that an accelerating charge emits electromagnetic radiation. But it is also known that the formula for the energy emitted as electromagnetic (EM) wave is, in general, not equal to the formula for the loss in the kinetic energy of the particle due to radiation compared to the situation when there is no radiation. This discrepancy has as yet remained unexplained. In the light of the proposed theory, this can be explained as follows: In addition to the energy expended by the external force, some kinetic energy is also released as the body accelerates and therefore shrinks in dimension. Therefore, the value of the kinetic energy loss is in general not equal to the energy loss due to radiation. The exact analysis of how a body accelerates under the influence of an external force is yet to be developed in the context of the proposed theory. 
Though both examples deal with electromagnetic emission by charged particles, the theory explained here doesn't make any distinction between charged and uncharged bodies and therefore even a neutral particle should emit EM wave when accelerated, albeit probably at a higher speed when, supposedly, a reasonable amount of its dimension is lost.

The two examples also lend an explanation to why an electron does not emit EM radiation when in orbit around the nucleus of an atom: the electron is traveling at a constant speed around the nucleus without an external source expending any energy for the purpose.

Also, it is seen that dimension-kinetic energy-electromagnetic energy and hence gravitationelectromagnetic energy are intimately related.

Since as per the proposed theory, all matter in accelerated motion, which implies almost all matter in the universe, emit EM radiation, the presence of CMB (Cosmic Microwave Background) radiation is also explained.

With regard to the above explanation a second example where the latent dimension of a body is lost is shown below:

Annihilation process: When matter and antimatter of masses $\mathbf{m}$ combine, the masses disappear and an electromagnetic wave with energy equal to $2 \mathbf{m c}^{2}$ is released. In the light of the theory presented the situation can be explained as follows: presumably the electromagnetic waves associated with the potent dimension of matter are out of phase with the electromagnetic waves associated with the corresponding dimension of antimatter whereas the electromagnetic waves associated with the latent dimensions of matter and antimatter are exactly in phase. Therefore, when they combine the EM waves corresponding to the latent dimensions of matter and antimatter with a combined energy of $2 \mathrm{mc}^{2}$ alone is released.

The proposed theory thus provides a convincing explanation to all major puzzles of contemporary physics without violating any established existing laws or theories including those proposed by Einstein. This fact, in my opinion, should be taken as a convincing proof of the correctness of the theory.

\section{IX.REFERENCES}

[1] Trimble, V. (1987), "Existence and nature of dark matter in the universe", Annual Review of Astronomy and Astrophysics. 25: 425-472.

[2] Bertone, G.; Hooper, D.; Silk, J. (2005). "Particle dark matter: Evidence, candidates and constraints", Physics Reports, 405 (5-6): 279-390.

[3] Peebles, P. J. E.; Ratra, Bharat (2003). "The cosmological constant and dark energy". Reviews of Modern Physics. 75 (2): 559-606. 
[4] 't Hooft, G. (2009), "Introduction to the Theory of Black Holes", Institute for Theoretical Physics / Spinoza Institute: $47-48$.

[5] Georgi, H.; Glashow, S.L. (1974), "Unity of All Elementary Particle Forces", Physical Review Letters. 32 (8): 438-41.

[6] Larmor J (1897), "LXIII: On the theory of the magnetic influence on spectra; and on the radiation from moving ions", Philosophical Magazine, 5. 44: 503-512.

[7] Griffiths, David J. (2013), Introduction to Electrodynamics (4th ed.), Boston, Mas.: Pearson.

[8] Albert Einstein (1905) "Zur Elektrodynamik bewegter Körper", Annalen der Physik 17: 891; English translation On the Electrodynamics of Moving Bodies by George Barker Jeffery and Wilfrid Perrett (1923); Another English translation On the Electrodynamics of Moving Bodies by Megh Nad Saha (1920).

[9] Albert Einstein (2001), Relativity: The Special and the General Theory (Reprint of 1920 translation by Robert W. Lawson ed.), Routledge, p. 48.

[10] Edwin F. Taylor \& John Archibald Wheeler (1992), Spacetime Physics: Introduction to Special Relativity, W. H. Freeman. 